\title{
A functional magnetic resonance imaging investigation of the autonomous sensory meridian response
}

\author{
Stephen D Smith ${ }^{\text {Corresp., } 1}$, Beverley K. Fredborg ${ }^{2}$, Jennifer Kornelsen ${ }^{3}$ \\ ${ }^{1}$ Department of Psychology, University of Winnipeg, Winnipeg, Manitoba, Canada \\ 2 Department of Psychology, Ryerson University, Toronto, Ontario, Canada \\ 3 Department of Radiology, University of Manitoba, Winnipeg, Manitoba, Canada \\ Corresponding Author: Stephen D Smith \\ Email address: s.smith@uwinnipeg.ca
}

Background: Autonomous Sensory Meridian Response (ASMR) is a sensory-emotional experience in which specific stimuli (ASMR 'triggers') elicit tingling sensations on the scalp, neck, and shoulders; these sensations are accompanied by a positive affective state. In the current research, functional magnetic resonance imaging (fMRI) was used in order to delineate the neural substrates of these responses. Methods: Seventeen individuals with ASMR and 17 age- and sex-matched control participants underwent fMRI scanning while watching six four-minute videos. Three of the videos were designed to elicit ASMR tingling and three videos were not. Results: The results demonstrated that ASMR videos have a distinct effect on the neural activity of individuals with ASMR. The contrast of ASMR participants' responses to ASMR videos showed greater activity in the cingulate gyrus as well as in cortical regions related to audition, movement, and vision. This activity was not observed in Control participants. The contrast of ASMR and Control participants' responses to ASMR-eliciting videos detected greater activity in right cingulate gyrus, right paracentral lobule, and bilateral thalamus in ASMR participants; control participants showed greater activity in the lingual and culmen of the cerebellum. Conclusions: Together, these results highlight the fact that ASMR videos elicit activity in brain areas related to sensation, emotion, and attention in individuals with ASMR, but not in matched control participants. 
3

\section{A functional magnetic resonance imaging investigation of the autonomous sensory} meridian response

$9{ }^{1}$ Department of Psychology, University of Winnipeg, 515 Portage Avenue, Winnipeg, Manitoba, 10 Canada, R3B 2E9. Phone: (204)786-9737. Email: s.smith@uwinnipeg.ca

$11{ }^{2}$ Department of Psychology, Ryerson University, 350 Victoria Street, Toronto, Ontario, Canada, 12 M5B 2K3. Email: bfredborg@psych.ryerson.ca

13 3Department of Radiology, University of Manitoba, SR226 Kleysen Institute for Advanced

14 Medicine, 710 William Avenue, Winnipeg, Manitoba, Canada, R2H 2A6. Phone: (204) $787-$

15 5658. E-mail: Jennifer.Kornelsen@umanitoba.ca

*Corresponding Author

\section{Paper Statistics}

Word count (including references): 5333

Figures: 5

Tables: 3

References: 36 
Background: Autonomous Sensory Meridian Response (ASMR) is a sensory-emotional experience in which specific stimuli (ASMR 'triggers') elicit tingling sensations on the scalp, neck, and shoulders; these sensations are accompanied by a positive affective state. In the current research, functional magnetic resonance imaging (fMRI) was used in order to delineate the neural substrates of these responses. Methods: Seventeen individuals with ASMR and 17 age- and sex-matched control participants underwent fMRI scanning while watching six fourminute videos. Three of the videos were designed to elicit ASMR tingling and three videos were not. Results: The results demonstrated that ASMR videos have a distinct effect on the neural activity of individuals with ASMR. ASMR participants' responses to ASMR videos showed greater activity in the cingulate gyrus as well as in cortical regions related to audition, movement, and vision. This activity was not observed in Control participants. The contrast of ASMR and Control participants' responses to ASMR-eliciting videos detected greater activity in right cingulate gyrus, right paracentral lobule, and bilateral thalamus in ASMR participants; control participants showed greater activity in the lingula and culmen of the cerebellum. Conclusions: Together, these results highlight the fact that ASMR videos elicit activity in brain areas related to sensation, emotion, and attention in individuals with ASMR, but not in matched control participants. 
52 limbs - during the perception of specific auditory or audiovisual stimuli (Barratt \& Davis, 2015).

53 These "tingles" are often accompanied by a calming feeling that many individuals report as

54 being emotionally positive (Poerio, Blakey, Hostler, \& Veltri, 2018). Previous survey research

55 of individuals with ASMR has noted that the most common "ASMR triggers" are whispering,

56 low-pitched repetitive noises such as tapping sounds, and videos depicting socially intimate

57 situations (e.g., having one's hair brushed; Barratt \& Davis, 2015; Barratt, Spence, \& Davis, 2017). Although several studies have attempted to identify demographic or personality factors that might make an individual more likely to experience these atypical sensory experiences (Fredborg, Clark, and Smith, 2017, 2018; Janik McErlean \& Banissy, 2017), much less is known about the neural underpinnings of ASMR. In the current research, functional magnetic resonance imaging (fMRI) was used to examine how the neural responses to ASMR-eliciting videos differ between individuals with ASMR and a matched control group. between the brains of individuals with ASMR and matched controls. Using resting-state fMRI, Smith, Fredborg, and Kornelsen (2017) found that the functional connectivity of the default mode network (DMN; Raichle, 2015; Raichle, MacLeod, Snyder, Powers, Gusnard, \& Shulman, 2001) differed between ASMR and control populations. ASMR participants showed reduced functional connectivity in the right precuneus and posterior cingulate, the left medial frontal gyrus and thalamus, and both the left and right superior temporal gyri. Increased functional connectivity was observed in right occipital and left frontal cortical areas. The fact that 
72 traditional nodes of the DMN show weaker functional connectivity in ASMR - and that brain

73 regions outside of typical DMN nodes were recruited into the DMN—suggests that ASMR is

74 associated with the blending of several resting-state networks in the brain. Such a finding is

75 consistent with other studies of atypical sensory experiences including synesthesia (Dovern,

76 Fink, Fromme, Wohlschläger, Weiss, Riedl, 2012; Tomson, Narayan, Allen, \& Eagleman,

77 2013), the experience of auditory hallucinations, (Alderson-Day et al., 2015), and the subjective

78 perceptual effects associated with hallucinogenic drugs (Roseman, Leech, Fielding, Nutt, \&

79 Carhart-Harris, 2014).

A recent psychophysiological study has taken the investigation of ASMR one step further

by measuring autonomic nervous system responses during the ASMR experience itself (Poerio,

Blakey, Hostler, \& Veltri, 2018). Poerio and colleagues found that viewing ASMR-eliciting

videos led to an increase in skin conductance responses (SCR) and a decrease in heart rate in

individuals with ASMR, but not in control participants. These results are curious in that

increased SCRs are typically associated with physiological arousal (Boucsein, 1992) whereas a

slowed heart rate is associated with the opposite (e.g., Johnston \& Anastasiades, 1990; Shapiro,

Jamner, Goldstein, \& Delfino, 2001). The researchers concluded that these seemingly

contradictory results are related to the complexity of the ASMR experience. Self-report studies

has consistently shown that ASMR is associated with a feeling of calm (Barratt \& Davis, 2015;

Fredborg et al., 2017). However, the psychophysiological data suggest that ASMR is a

94 Kaernbach, 2011; Grewe, Kopiez, \& Altenmüüller, 2009). Indeed, survey research indicates that 
95

96

97 98

over $90 \%$ of individuals with ASMR view this experience as being different from frisson (Fredborg et al., 2018). Thus, it appears that the subjective positive emotions associated with ASMR co-occur with physiological responses in a manner that is both subjectively and biologically distinct from similar phenomena.

The purpose of the current study is to identify brain areas associated with the ASMR experience. Doing so will provide insights into the neurophysiological underpinnings of this phenomenon, thus allowing researchers to see how ASMR affects somatosensation and emotion rather than listing or describing those experiences. To date, only one fMRI study has measured brain activity during ASMR (Lochte, Guillory, Richard, \& Kelley, 2018). Lochte and colleagues presented five different seven-minute ASMR-eliciting videos to ten individuals with ASMR while they underwent an fMRI scan. A region-of-interest (ROI) analysis indicated that the experience of ASMR tingles was associated with increased activity in the nucleus accumbens, dorsal anterior cingulate gyrus, supplementary motor area, and a region including the insula and inferior frontal gyrus. More conservative whole-brain analyses noted activity in parts of the medial prefrontal cortex, insula, and nucleus accumbens. Although this initial study provided an important window into the neural substrates of ASMR, the authors also noted several limitations. These included the lack of a control group that did not experience ASMR as well as a small sample size $(\mathrm{n}=10)$.

The current research builds upon the research of Lochte and colleagues by testing a larger number of participants and including a control group of ASMR-insensitive individuals. Individuals with ASMR and age- and sex-matched control participants viewed three popular YouTube.com videos designed to elicit ASMR tingles, as well as three control videos (i.e., videos that do not trigger ASMR tingles) of similar complexity. This design allowed us to 
118 corroborate the results of Lochte and colleagues (2018) in ASMR participants while also

119 demonstrating that similar neural changes do not occur in individuals who do not experience

120 ASMR. Although the current research was performed prior to the publication of Lochte and

121 colleagues' work, we also hypothesized that ASMR would be associated with increased activity

122 in regions related to emotional responses including the anterior cingulate cortex, nucleus

123 accumbens, and hypothalamus. The functions of these brain areas are consistent with the

124 subjective reports of the ASMR experience, with anterior cingulate activity being related to both

125 attentional control and emotion-attention interactions (Gasquoine, 2013), the nucleus accumbens

126 being involved with reward responses (Floresco, 2015), and the hypothalamus being central to

127 hormone release and embodied emotional responses (e.g., Craig, 2002). We also speculated that

128 ASMR would be associated with increased activity in sensorimotor regions (i.e., precentral and

129 postcentral gyri). This expectation was based both upon the descriptions of the tingling

130 sensations as reported in previous studies of ASMR (Barratt \& Davis, 2015; Fredborg et al.,

131 2017), as well as the results of previous neuroimaging investigations showing that positive

132 emotional responses to auditory stimuli — particularly music — also activated sensorimotor

133 regions of the cortex (e.g., Kleber, Birbaumer, Veit, Trevorrow, \& Lotze, 2007; see Koelsch \&

134 Stegemann, 2012, and Schaefer, 2017, for reviews).

\section{Methods}

\section{Participants}

The participants consisted of 17 individuals with ASMR ( 8 males, 9 females; $M_{\text {age }}=$

$\left.13822.71 ; S D_{\text {age }}=4.74\right)$ and 17 age $(+/-3$ years $)$ and sex-matched control participants ( 8 males, 9

139 females; $M_{\text {age }}=22.76 ; S D_{a g e}=5.39$ ). This sample size was based on published calculations for 
140 neuroimaging studies (Desmond \& Glover, 2002; Hayasaka et a., 2007) The ASMR

141 participants were recruited from the Winnipeg, Manitoba, Canada community via social media

142 posts and word-of-mouth. In order to confirm that participants did in fact have ASMR,

143 participants were asked to view two ASMR-related YouTube.com videos while in the presence

144 of the second author. All participants confirmed that the videos triggered tingling sensations.

145 The participants also provided information about their ASMR history and completed an ASMR

146 checklist (Fredborg et al., 2017) which outlines various triggers that typically elicit tingling

147 sensations in individuals with ASMR.

Controls participants were recruited from the University of Winnipeg student population.

149 These participants also viewed the ASMR-related videos to ensure that they did not experience ASMR. Moreover, these participants were interviewed by the same investigator to determine

151

152

153

154

155

156

157

that they had never experienced ASMR. The data from two participants were removed due to excessive movement in the scanner and were replaced by new participants; the demographic data reported above reflect the ages of the sample used in our analyses.

None of the ASMR or control participants reported any history of neurological injury or psychiatric illness. Informed, written consent was provided by all participants prior to entering the MRI scanner. Participants also underwent MR safety screening prior to scanning. This research received ethical approval from the research ethics boards (REBs) of both the University of Manitoba Bannatyne Campus and the University of Winnipeg (Ethical Application Ref: HS18236; B2014:078). All participants received a remuneration of \$50 CAD (approximately $\$ 40$ USD). 
162 Procedure

163

164

165

166

167

168

169

170

171

172

173

174

175

176

177

178 179 alternated).

180

181

182

After providing informed, written consent and undergoing MR safety screening,

participants were brought into the MRI suite. All scans began with an initial localizer image and shimming, followed by a 7-minute structural MRI scan. Participants then completed six fMRI runs. Each run lasted five minutes and 15 seconds and consisted of a one-minute presentation of a fixation cross, a four-minute presentation of a video, and a 15 -second presentation of a fixation cross. The sound for the videos was presented via speakers in the MRI suite; all participants reported that they were able to clearly hear the six videos.

The six videos presented during this study included three videos designed to elicit ASMR tingles (hereafter "ASMR-related videos") and three videos that were not likely to elicit ASMR (hereafter "control videos"). The ASMR-related videos all involved whispering and viewing a socially emotional scene (the application of make-up, a simulated lice check, or an individual brushing someone's hair). The control videos involved normal-volume speech and were on the topics of studying suggestions, gardening tips, and make-up application instructions. The order of the three ASMR-related videos and the three control videos was randomized across participants; however, the fMRI runs were presented such that participants did not view two ASMR-related or two control videos in a row (i.e., ASMR-related and control videos were

After completing the experimental session, participants indicated whether they experienced ASMR during the viewing of any of the six fMRI runs and, if so, the intensity (out of 10, with 10 representing the highest intensity of tingles they had ever experienced) of the 
183 resulting ASMR tingles. Importantly, participants were not told in advance which videos were 184 expected to elicit ASMR.

185

186

187

\section{Data Acquisition}

Data were acquired using a 3-Tesla Siemens TRIO MRI scanner (Siemens, Erlangen,

Germany). Anatomical data were acquired with high-resolution T1-weighted gradient-echo images using a magnetization-prepared rapid-gradient-echo (MP-RAGE) sequence with the following parameters: slice thickness $=1 \mathrm{~mm}$; distance factor $=50 \%$;R $=1900 \mathrm{~ms} ; \mathrm{TE}=$ $2.99 \mathrm{~ms}$; in-plane resolution $=1.0 \times 1.0$; matrix $=256 \times 256$; and field of view $(\mathrm{FOV})=250$ $\mathrm{mm}$.

Functional MRI data acquisition consisted of a 5 minute 15 second scan using a wholebrain echo-planar imaging (EPI) sequence with the following parameters: slick thickness $=3$ $\mathrm{mm}$; distance factor $=0 \% ; \mathrm{TR}=3000 \mathrm{~ms} ; \mathrm{TE}=30 \mathrm{~ms}$; flip angle $=90^{\circ}$; matrix $=64 \mathrm{X} 64 ;$ and $\mathrm{FOV}=240 \mathrm{~mm}$.

\section{Data Analysis}

The post-scan ratings of ASMR-related videos by the ASMR participants indicated that some individuals did not experience tingles in response to some of the videos. However, all ASMR participants did indicate experiencing tingles in response to at least two of the three ASMR-related videos. Therefore, for each participant, we analyzed the data from two out of the three fMRI runs involving the presentation of ASMR-related videos. If a participant reported experiencing tingles in response to all three ASMR-related videos, we selected the two fMRI runs linked with the highest tingle intensity ratings for that participant. No participants experienced ASMR-related tingles to any of the control videos. The control-video fMRI runs 
205

206

207

208

209

210

211

212

213

214

215

216

217

218

219

220

221

222

223

224

225

226

227

were yoked to the particular ASMR-related video fMRI runs used for the analyses. For example, if an ASMR participant rated ASMR videos 1 and 3 as eliciting the most intense tingles, then control videos 1 and 3 were used for that participant's within-subjects contrast.

For the control participants, we analyzed the data from the two runs used for the ASMR participant to whom they were matched. For example, if an ASMR participant reported experiencing high-intensity tingles in response to ASMR-related videos 2 and 3, then the fMRI runs for ASMR-related videos 2 and 3 were used when analyzing the data for the control participant who was age- and sex-matched with that ASMR participant.

Imaging data were preprocessed and analyzed in BrainVoyager QX software (Brain Innovation, BV, Maastricht, The Netherlands). Preprocessing of functional data included slice scan time correction, 3D motion correction, linear trend removal, and temporal high pass filtering. The functional data were coregistered to the anatomical data, and were warped to standardized Talairach space. The time-series data were dummy-coded to account for multiple runs per participant. The stimulation protocol was created to represent the timing of the video presentation and baseline. Individual data were analyzed with a single subject GLM. These were entered into a multi-study multi-subject GLM and compiled for group analysis. A random effects ANCOVA was run. The F-test interaction was obtained followed by contrasts of interest; specifically [11-1-10000] to address the within-subjects effect of the ASMR-related video compared to the control video in ASMR participants, [000011-1-1] to address the within-subjects effect of the ASMR-related video compared to the control video in control participants, and [1100-1-100] to address the between groups difference of ASMR participants compared to control participants while watching ASMR-related videos. Beta values were extracted and plotted for the contrasts of interest. The within-subjects results were displayed at $p<0.01$ and 
228 corrected for multiple comparisons using a cluster threshold estimator. The interaction and

229 between-subjects results were displayed at $p<0.05$ and a cluster threshold of 10 voxels was

230 applied. Clusters were converted to volumes-of-interest, and peak intensity coordinates, $t$-value,

$231 p$-value, and voxel counts were given for each cluster. The peak intensity coordinates were

232 entered in Talairach-Client software (http://www.talairach.org/client.html) which produced the

233 anatomical labels and Brodmann Areas (BA), if applicable.

\section{Results}

235

The statistical test for the interaction effect revealed significant activity in bilateral

236

237

238

239

240

241

242

243

244

245

246

247

248

249

250

superior parietal (Brodmann area (BA) 7), in the right paracentral lobule (BA5) and cerebellum, and in the left cingulate gyrus (BA 32), precuneus (BA 7), inferior and medial frontal gyri (BA 9), and precentral gyrus (BA6). The results are displayed in Figure 1 and the peak coordinates for these regions are shown in Table 1.
[Please insert Figure 1 here.]
[Please insert Table 1 here.]

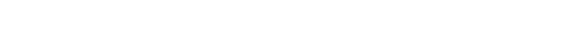

Results of the within-groups contrasts are listed in Table 2 and displayed in Figure 2 and

Figure 3. A within-groups contrast was performed comparing the neural responses of ASMR participants when they viewed ASMR-related or control videos. This contrast detected significant increases in activity in the right superior frontal gyrus (BA) 8), right dorsal anterior cingulate gyrus (BA 32), and right precentral gyrus (BA 4). Significant clusters were detected in the left hemisphere in the medial frontal (BA 6), precentral (BA 6), and superior temporal (BA 22) gyri, as well as in the cuneus (BA 18). Thus, ASMR videos elicited greater activity in frontal-lobe regions as well as in areas related to vision, audition, and sensorimotor responses. 
251 The identical analysis (i.e., responses to ASMR-related or control videos) was performed using

252 the data from control participants. This contrast yielded one significant difference: a decrease in 253 activity in the right cuneus (BA 18).

254

255

256

257

258

259

260

261

262

263

264

265

266

267

268

269

270

271

272

273
[Please insert Table 2 here.]

[Please insert Figure 2 here.]

[Please insert Figure 3 here.]

participants during the viewing of ASMR-related videos. The coordinate and statistical details

for the significant results are listed in Table 3 and displayed in Figures 4 and 5. This contrast

demonstrated that ASMR-related videos elicited greater activity in ASMR participants in the

right paracentral lobule (BA 5), right anterior cingulate cortex (BA 24), left precuneus (BA 7), as

well as bilateral activity in the thalamus (Figure 4 and Figure 5). Decreased activity was

observed in ASMR participants in the right lingula and the left culmen of the cerebellum.

[Please insert Table 3 here.]

[Please insert Figure 4 here.]

[Please insert Figure 5 here.]

\section{Discussion}

The current results demonstrate that ASMR has both emotional and sensorimotor characteristics. ASMR-related videos elicited activity in numerous brain areas in individuals with ASMR including medial prefrontal brain areas, the precentral gyrus, left superior temporal 
274 gyrus, and left cuneus. The same statistical contrast (ASMR videos $>$ control videos) only

275 elicited a decrease in activity in the right cuneus in control participants. Additionally, a

276 comparison of responses to the ASMR videos showed greater activity in ASMR than control

277 participants in the thalamus, left precuneus, cingulate gyrus, and medial sensorimotor regions.

278 These data show some consistency with the previous neuroimaging study of Lochte and

279 colleagues (2018), namely activity in the anterior cingulate gyrus and motoric regions of the

280 cortex; however, some of the results did differ across studies, including the lack of nucleus

281 accumbens activity in the current study. In this Discussion, we will examine the notable regions

282 of activity detected in the current study while also highlighting avenues for future neuroimaging

283 studies of ASMR.

284 The results of the current study highlight the sensorimotor nature of ASMR. Individuals

285 with ASMR demonstrated greater activity in both the precentral (i.e., the motor cortex) and

286 postcentral gyri (i.e., the somatosensory cortex) during the viewing of ASMR-related videos than

287 when watching videos that did not elicit tingling sensations. Additionally, ASMR participants

288 showed greater activity in the paracentral lobule - the continuation of the precentral and

289 postcentral gyri on the medial aspect of the brain — during the perception of ASMR-related

290 videos than did control participants. Together, these data demonstrate that the tingling

291 sensations elicited by ASMR triggers are not psychosomatic; rather, they are associated with

292 increased activity in the sensorimotor regions of the cortex.

293 The enhanced activity in the visual and auditory cortices may be linked with the affective

294 nature of the ASMR videos. Previous neuroimaging research has reported increased activity in

295 visual areas during the perception of emotional stimuli (e.g., Morris et al., 1998; Surguladze et

296 al., 2003). Given that ASMR-related videos elicit tingling in ASMR participants, these stimuli 
297 are likely more salient and emotionally arousing than control videos. Such a difference in

298 perceived salience and arousal may explain the activity detected in the cuneus during the ASMR-

299 related video vs. control video contrast and in the precuneus during the contrast of ASMR and

300 control participants' responses to ASMR-related videos. Unfortunately, the ASMR participants'

301 ratings of the intensity of their tingling responses did not provide much variability, thus limiting

302 our ability to examine this hypothesis. This hypothesis could be tested in future research by

303 asking participants to provide intensity ratings at multiple times within each fMRI run. This

304 increase in measurements would also provide additional statistical power for analyses.

305

306

307

308

309

310

311

312

313

314

315

316

317

318

319
An additional potential explanation for the observed activity in the auditory cortex relates

to the acoustic qualities of the ASMR videos themselves. Barratt and colleagues (2017) found

that ASMR tingles were most likely to be elicited by lower-pitched, complex sounds. It is

possible that the increase in superior temporal lobe activity observed when ASMR participants

listened to ASMR videos could be related to an enhanced sensitivity to these low-frequency

sounds. This possibility could be tested by manipulating the acoustic characteristics of the test

stimuli. Unfortunately, the current study, which only included data from two ASMR-video runs

and two control-video runs for each participant, did not have enough variability in ASMR

triggers to test this possibility.

The observed activity in the dorsal anterior cingulate gyrus is more difficult to classify, as

this region is involved with a number of cognitive and affective functions. The significant

clusters in the current study included Brodmann areas 24 and 32. These regions are part of the

cognitive/attentional region of the anterior cingulate typically involved in attentional control

(Bush, Luu, \& Posner, 2000). This activity seems reasonable given that ASMR-relevant stimuli

would likely be quite engaging to individuals with ASMR. Moreover, it is possible that the

PeerJ reviewing PDF | (2019:01:34028:1:1:NEW 29 Apr 2019) 
320 observed activity in the dACC may be related to its membership in the salience network-a

321 network that functions as a switch between networks involved in internal mentation (default

322 mode network) and attentional processes (central executive network) (Goulden, et al., 2014).

323 Given that the ASMR experience involves an attention to an external stimulus which evokes an

324 internal sensation, it is possible that the dACC facilitates this experience in ASMR. Importantly,

325 this activity is consistent with the dorsal anterior cingulate activity reported by Lochte and

326 colleagues (2018). The Montreal Neurological Institute coordinates reported in that study (-2,

32724,38 ) translate into Brodmann area 8, a medial region slightly above the activity observed in

328 the current study. Thus, there appears to be inter-study consistency related to ASMR in this

329 brain region. It is also worth considering, however, that this region of the anterior cingulate

330 cortex also has reciprocal connections with the more ventral, affective regions of this structure

331 (Hamani, Mayberg, Stone, Laxton, Haber, \& Lozano, 2011; Ongur \& Price, 2000). Therefore, it

332 is premature to conclude that this anterior cingulate activity is exclusively attentional in nature.

333 The contrast comparing neural responses of ASMR and control participants during the

334 viewing of ASMR-related videos also detected bilateral activity in the thalamus. Given that the

335 thalamus serves as a "sensory relay station" for visual, auditory, and somatosensory information

336 (e.g., Guillery \& Sherman, 2002; Sherman, 2017) — the sensory modalities typically associated

337 with ASMR - the enhanced activity in this region in ASMR participants implies that atypical

338 sensory integration is likely occurring at this very early stage of sensory processing. This

339 integration could take the form of thalamic nuclei that typically process stimuli from one sensory

340 modality (e.g., somatosensation) becoming active in response to additional modalities (e.g.,

341 audition), perhaps due to overlapping synaptic projections between nuclei. It is also possible that 
342 the enhanced thalamic activity was a result of hypersensitivity in a specific thalamic nucleus

343 (e.g., the pulvinar). Additional research is needed to test these possibilities.

The atypical thalamic activity noted in the current research is consistent with a resting-

345 state fMRI study comparing ASMR and control participants which showed inter-group

346 differences in the functional connectivity of the thalamus (Smith et al., 2017). It also

347 complements an unusual case study of a patient who acquired a form of sensory-emotional

348 synesthesia following a thalamic stroke (Schweizer et al., 2013). This patient did not experience

349 synesthesia prior to his infarct; however, during neurorehabilitation, he discovered that he

experienced numerous anomalous sensory associations, including sensory-emotional synesthesia.

Most notable among these was his response to brass musical instruments (particularly the brass music from older James Bond movies), which he said led to an embodied experience that was quite pleasurable. Although it is unclear whether this patient experiences ASMR per se, the phenomenological similarity between his post-stroke sensations and ASMR is noteworthy and suggests that atypical thalamic connectivity may underlie both phenomena.

A particularly surprising result from the current study was the lack of activity in reward centers such as the nucleus accumbens. This region was active in Lochte and colleagues (2018) ROI analysis of 10 individuals with ASMR. This difference may be due to the fact that several of the videos used in the previous study involved the actors touching each other (e.g., a grooming video) or to the fact that the current study measured brain activity across the entire 4-minute block of the video rather than segmenting it based on participants' self-reports of tingling. In future studies, it would be useful to combine psychophysiological measurements and self-reports during the acquisition of fMRI data. This combined methodology would allow us to more thoroughly map the central- and peripheral-nervous-system responses to ASMR triggers. 


\section{Limitations and Future Directions}

Although the current research provides novel information about the neural responses of individuals with and without ASMR to videos designed to elicit ASMR, its shortcomings also highlight avenues for future research. Survey studies of individuals with ASMR note that whispering, tapping, and binaural sounds are common triggering stimuli (Barrett et al, 2015, 2017; Fredborg et al., 2017). Thus, it would be informative to have ASMR participants and matched controls perceive these different types of stimuli while in the fMRI suite to determine whether the neural responses during ASMR experiences differ across trigger types. It would also be interesting to examine whether responses differ when socially intimate triggering stimuli (e.g., grooming videos) involve a first- versus a third-person perspective. A third limitation relates to how the subjective intensity of the ASMR tingles were measured. It is unclear whether the selfreport ratings of tingle intensity are comparable across participants (i.e., is unclear whether a 5/10 rating for one participant reflects the same ASMR intensity as a 5/10 rating from another participant). Future studies should acquire psychophysiological measures such as skin conductance responses and heart rate in order to provide a more valid measure of ASMR intensity (see Poerio et al., 2018). Finally, using a more temporally sensitive neuroimaging technique such as electroencephalography (EEG) or magnetoencephalography (MEG) would allow researchers to more precisely measure the time course of activity during the ASMR experience; specifically, these techniques could measure the location and frequency of neuronal activity immediately prior to and following the onset of ASMR tingles. Therefore, although the current study and the work of Lochte and colleagues (2018) provide complementary insights into the neural architecture of ASMR, there is a clear need for additional neuroimaging research in order to delineate the neural substrates of this atypical sensory-emotional experience. 
390

391

392

393

394

395

396

397

398

399

400

\section{Conclusions}

The results of the current study highlight the complexity of the ASMR experience. When viewing ASMR-eliciting videos, individuals with ASMR showed increases in neural activity in regions of the cortex related to attention, audition, emotion, and movement. This activity was not observed in control participants. When responses of ASMR and control participants viewing ASMR videos were compared, individuals with ASMR showed greater activity in the thalamus, anterior cingulate cortex, precuneus and medial sensorimotor regions. Together, these analysis demonstrate that ASMR is not simply a sensory or an emotional phenomenon. Instead, the data suggest that ASMR involves sensory, motoric, affective, and attentional components. 


\section{Acknowledgements}

402 The authors wish to thank Teresa Figley and the radiology technical staff for their assistance

403 with data collection.

404

405 
408

409

410

411

412

413

414

415

416

417

418

419

420

421

422

423

424

425

426

427

428

429

Alderson-Day, B., McCarthy-Jones, S., \& Fernyhough, C. (2015). Hearing voices in the resting brain: A review of intrinsic functional connectivity research on auditory verbal hallucinations. Neuroscience and Biobehavioral Reviews, 55, 78-87. doi: 10.1016/j.neubiorev.2015.04.016

Barratt, E. L., \& Davis, N. J. (2015). Autonomous Sensory Meridian Response (ASMR): a flowlike mental state. PeerJ, 3, e851. doi: 10.7717/peerj.851

Barratt, E. L., Spence, C., \& Davis, N. J. (2017) Sensory determinants of the autonomous sensory meridian response (ASMR): understanding the triggers. PeerJ, 5, e3846. doi: 10.7717/peerj.3846

Benedek, M., \& Kaernbach, C. (2011). Physiological correlates and emotional specificity of human piloerection. Biological Psychology, 86, 320-329. doi: 10.1016/j.biopsycho.2010.12.012

Boucsein, W. (1992). Electrodermal Activity. New York: Plenum Press.

Bush, G., Luu, P., \& Posner, M. I. (2000). Cognitive and emotional influences in anterior cingulate cortex. Trends in Cognitive Sciences, 4, 215-222. doi: 10.1016/S13646613(00)01483-2

Craig, A.D. (2003). Interoception: the sense of the physiological condition of the body. Current Opinions in Neurobiology, 13, 500-505. doi: 10.1016/S0959-4388(03)00090-4

del Campo, M. A., \& Kehle, T. J. (2016). Autonomous sensory meridian response (ASMR) and frisson: Mindfully induced sensory phenomena that promote happiness. International Journal of School \& Educational Psychology, 4, 99-105. doi:

$10.1080 / 21683603.2016 .1130582$ 
431

432

433

434

435

436

437

438

439

440

441

442

443

444

445

446

447

448

449

450

451

Desmond, J.E. \& Glover, G.H. (2002). Estimating sample size in functional MRI (fMRI) neuroimaging studies: statistical power analyses. Journal of Neuroscience Methods, 118, 115-128. doi: 10.1016/S0165-0270(02)00121-8

Dovern, A., Fink, G. R., Fromme, A. C., Wohlschläger, A. M., Weiss, P. H., Riedl, V. (2012). Intrinsic network connectivity reflects consistency of synesthetic experiences. Journal of Neuroscience, 32, 7614-7621. doi: 10.1523/JNEUROSCI.5401-11.2012.

Floresco, S. B. (2015). The nucleus accumbens: an interface between cognition, emotion, and action. Annual Review of Psychology, 66, 25-52. doi: 10.1146/annurev-psych-010213115159.

Fredborg, B. K., Clark, J., \& Smith, S. D. (2017). The big five personality traits and Autonomous Sensory Meridian Response (ASMR). Frontiers in Psychology, 8, 247. doi:10.3389/fpsyg.2017.00247

Fredborg, B. K., Clark, J. M., \& Smith, S. D. (2018). Mindfulness and autonomous sensory meridian response (ASMR). PeerJ, 6, e5414. doi: 10.1016/j.finel.2017.07.004

Gasquoine, P. G. (2013). Localization of function in anterior cingulate cortex: from psychosurgery to functional neuroimaging. Neuroscience and Biobehavioral Reviews, 37, 340-348. doi: 10.1016/j.neubiorev.2013.01.002.

Goulden, N., Khusnulina, A., Davis, N.J., Bracewell, R.M., Bokde, A.L., McNulty, J.P., \& Mullins, P.G. (2014). The salience network is responsible for switching between the default mode network and the central executive network: replication from DCM. NeuroImage, 99, 180-190. doi: 10.1016/j.neuroimage.2014.05.052 
452 Grewe, O., Kopiez, R., \& Altenmüüller, E. (2009). The chill parameter: Goose bumps and

453 shivers as promising measures in emotion research. Music Perception: An

454 Interdisciplinary Journal, 27, 61-74. doi: 10.1525/mp.2009.27.1.61

455 Guillery, R. W., \& Sherman, S. M. (2002). Thalamic relay function and their role in cortico-

456

457

458

459

460

461

462

463

464

465

466

467

468

469

470

471

472

473

474 cortical communication: generalizations from the visual system. Neuron, 33, 163-175. doi: 10.1016/S0896-6273(01)00582-7

Hamani, C., Mayberg, H., Stone, S., Laxton, A., Haber, S., \& Lozano, A. M. (2011). The subcallosal cingulate gyrus in the context of major depression. Biological Psychiatry, 69, 301-308. doi:10.1016/j.biopsych.2010.09.034

Hayasaka, S., Peiffer, A.M., Hugenschmidt, C.E., Laurienti, P.J. (2007). Power and sample size calculation for neuroimaging studies by non-central random field theory. NeuroImage, 37, 721-730.

Janik McErlean, A., \& Banissy, M. (2017). Assessing individual variation in personality and empathy traits in self-reported Autonomous Sensory Meridian Response. Multisensory Research, 30, 601-613. doi:10.1163/22134808-00002571

Johnston, D. W., \& Anastasiades, P. (1990). The relationship between heart rate and mood in real life. Journal of Psychosomatic Research, 34, 21-27.

Kleber, B., Birbaumer, N., Veit, R., Trevorrow, T., and Lotze, M. (2007). Overt and imagined singing of an Italian aria. NeuroImage, 36, 889-900. doi: 10.1016/j.neuroimage.2007.02.053

Koelsch S., Stegemann T. (2012). The brain and positive biological effects in healthy and clinical populations (pp. 436-456). In R. MacDonald, D. Kreutz, and L. Mitchell (eds.), Music, Health and Wellbeing. Oxford: Oxford University Press. 
475 Lochte, B. C., Guillory, S. A., Richard, C. A. H., \& Kelley, W. M. (2018). An fMRI

476 investigation of the neural correlates underlying the autonomous sensory meridian

477 response (ASMR). BioImpacts, 8, 295-304. doi: 10.15171/bi.2018.32

478 Morris, J. S., Friston, K. J., Buchel, C., Frith, C. D., Young, A. W., Calder, A. J., \& Dolan, R. J.

479

480

481

482

483

484

485

486

487

488

489

490

491

492

493

494

495

496

497

(1998). A neuromodulatory role for the human amygdala in processing emotional facial expressions. Brain, 121, 47-57.

Ongur, D., Price, J. L. (2000): The organization of networks within the orbital and medial prefrontal cortex of rats, monkeys and humans. Cerebral Cortex, 10, 206 -219. doi: $10.1093 /$ cercor/10.3.206

Poerio, G. L., Blakey, E., Hostler, T. J., \& Veltri, T. (2018). More than a feeling: Autonomous sensory meridian response (ASMR) is characterized by reliable changes in affect and physiology. PLoS ONE, 13, e0196645. doi: 10.1371/journal.pone.0196645

Raichle, M. E. (2015). The brain's default mode network. Annual Review of Neuroscience, 38, 433-447. doi: 10.1146/annurev-neuro-071013-014030

Raichle, M. E., MacLeod, A. M., Snyder, A. Z., Powers, W. J., Gusnard, D. A., \& Shulman, G. L. (2001). A default mode of brain function. Proceedings of the National Academy of Sciences, 98, 676-682. doi: 10.1073/pnas.98.2.676

Roseman, L., Leech, R., Feilding, A., Nutt, D. J., \& Carhart-Harris, R. L. (2014). The effects of psilocybin and MDMA on between-network resting state functional connectivity in healthy volunteers. Frontiers in Human Neuroscience, 8, 204. doi: 10.3389/fnhum.2014.00204

Schaefer, H.-E. (2017). Music-evoked emotions: Current studies. Frontiers in Human Neuroscience, 11, 600. doi: 10.3389/fnins.2017.00600 
498 Schweizer, T. A., Li, L., Alexander, M. P., Smith, S. D., Graham, S., Fischer, C., \& Fornazarri, 499 L. (2013). From the thalamus with love: a rare window into the locus of emotional 500 synesthesia. Neurology, 51, 509-510. doi: 10.1212/WNL.0b013e31829d86cc.

501 Shapiro, D., Jamner, L. D., Golstein, I. B., \& Delfino, R. J. (2001). Striking a chord: moods, 502 blood pressure, and heart rate in everyday life. Psychophysiology, 38, 197-204. doi: 10.1111/1469-8986.3820197

504

505

506

507

508

509

510

511

512

513

514

515

Sherman, S. M. (2017). Functioning of circuits connecting thalamus and cortex. Comprehensive Physiology, 7, 713-739. doi: 10.1002/cphy.c160032

Smith, S. D., Fredborg, B. K., \& Kornelsen, J. (2017). An examination of the default mode network in individuals with Autonomous Sensory Meridian Response (ASMR). Social Neuroscience, 12, 361-365. doi:10.1080/17470919.2016.1188851

Surguladze, S. A., Brammer, M. J., Young, A. W., Andrew, C., Travis, M. J., Williams, S. C. R., \& Phillips, M. L. (2003). A preferential increase in the extrastriate response to signals of danger. NeuroImage, 19, 1317-1328. doi: 10.1016/S1053-8119(03)00085-5

Tomson, S. N., Narayan, M., Allen G. I., \& Eagleman, D. M. (2013). Neural networks of colored sequence synesthesia. Journal of Neuroscience, 33, 14098-15106. doi: 10.1523/JNEUROSCI.5131-12.2013. 


\section{Table $\mathbf{1}$ (on next page)}

Coordinate and statistical values for the interaction effect

Regions of significant differential activation were detected for the interaction between participant group (ASMR, control) and video type (ASMR-eliciting videos, control videos). 
1

Table 1. Coordinate and statistical values for the interaction effect.

\begin{tabular}{|c|c|c|c|c|c|c|c|}
\hline \multirow[b]{2}{*}{ Region label } & \multirow[b]{2}{*}{ Brodmann Area } & \multirow{2}{*}{$\begin{array}{l}\text { Cluster size } \\
\text { (\# voxels) }\end{array}$} & \multicolumn{3}{|c|}{ TAL coordinates } & \multirow[b]{2}{*}{ f-value } & \multirow[b]{2}{*}{ p-value } \\
\hline & & & $\mathbf{x}$ & $\mathbf{y}$ & $\mathbf{z}$ & & \\
\hline R Superior Parietal Lobule & 7 & 471 & 26 & -68 & 54 & 4.938689 & 0.003 \\
\hline R Cerebellum (culmen) & $*$ & 358 & 11 & -32 & -15 & 6.690252 & $<0.001$ \\
\hline R Paracentral Lobule & 5 & 697 & 2 & -38 & 51 & 9.731303 & $<0.001$ \\
\hline L Cingulate Gyrus & 32 & 536 & -1 & 16 & 39 & 5.823417 & 0.001 \\
\hline L Precuneus & 7 & 434 & -1 & -59 & 48 & 5.478245 & 0.002 \\
\hline L Medial Frontal Gyrus & 9 & 382 & -7 & 37 & 27 & 5.877485 & $<0.001$ \\
\hline L Superior Parietal Lobe & 7 & 631 & -29 & -68 & 51 & 5.822857 & 0.001 \\
\hline L Precentral Gyrus & 6 & 378 & -46 & -8 & 39 & 5.686726 & 0.001 \\
\hline L Inferior Frontal Gyrus & 9 & 400 & -43 & 4 & 27 & 5.402328 & 0.002 \\
\hline
\end{tabular}

2 


\section{Figure 1}

Activation from the interaction of participant group and video type

Regions of significant differential activation for the effect of the interaction between

participant group (ASMR, control) and video type (ASMR-eliciting, control videos) include

bilateral superior parietal, right cerebellum and paracentral lobule, and left cingulate, medial prefrontal, precentral, and inferior frontal gyri and precuneus. (A) Sagittal view. (B) Coronal view. (C) Transverse view. Results displayed at $p<0.05$, cluster threshold corrected at 10 voxels. $\mathrm{SAG}=$ sagittal, $\mathrm{COR}=$ coronal, $\mathrm{TRA}=$ transverse $\mathrm{A}=$ anterior, $\mathrm{P}=$ posterior, $\mathrm{R}=$ right, $L=$ left.

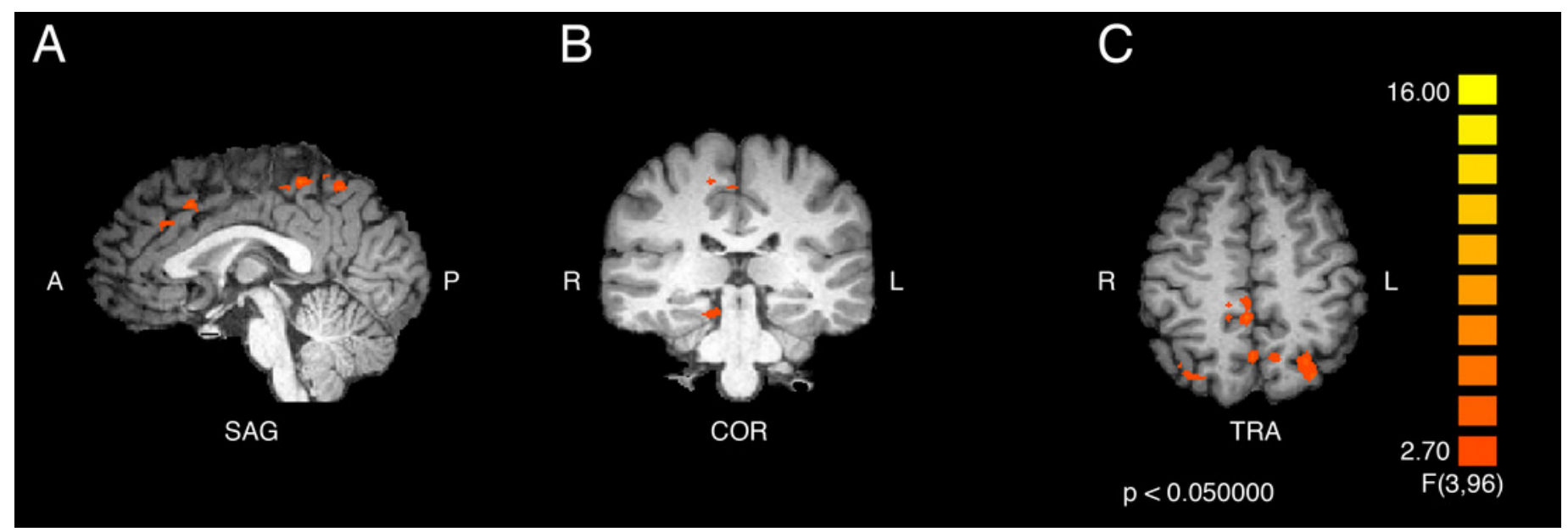




\section{Table 2 (on next page)}

Coordinate and statistical values for the contrast of ASMR-related videos > control (nonASMR- eliciting) videos, shown for ASMR participants and control participants.

For the contrast involving ASMR participants, the positive $t$-values indicate regions of increased activity for ASMR-eliciting videos as compared to the control videos. No significant decreases in activity were observed. For the contrast involving control participants, the negative $t$-value indicates the region of decreased activity for the ASMR-eliciting videos as compared to the control videos. No significant increases in activity were observed for the control participants. 
1 Table 2. Coordinate and statistical values for the contrast of ASMR-related videos $>$ control (non-ASMR2 eliciting) videos, shown for ASMR participants and control participants.

\begin{tabular}{|c|c|c|c|c|c|c|c|}
\hline \multirow[b]{2}{*}{ Region label } & \multirow[b]{2}{*}{ Brodmann Area } & \multirow{2}{*}{$\begin{array}{l}\text { Cluster size } \\
\text { (\# voxels) }\end{array}$} & \multicolumn{3}{|c|}{ TAL coordinates } & \multirow[b]{2}{*}{ t-value } & \multirow[b]{2}{*}{ p-value } \\
\hline & & & $\mathbf{x}$ & $\mathbf{y}$ & $\mathbf{z}$ & & \\
\hline \multicolumn{8}{|l|}{ ASMR participants } \\
\hline Right Precentral Gyrus & 4 & 343 & 50 & -8 & 42 & 3.949 & $<0.001$ \\
\hline Right Cingulate Gyrus & 32 & 864 & 5 & 7 & 39 & 4.411 & $<0.001$ \\
\hline Right Superior Frontal Gyrus & 8 & 359 & 8 & 46 & 42 & 3.84 & $<0.001$ \\
\hline Left Cuneus & 18 & 445 & -19 & -86 & 18 & 3.789 & $<0.001$ \\
\hline Left Medial Frontal Gyrus & 6 & 783 & -16 & -17 & 51 & 4.44 & $<0.001$ \\
\hline Left Precentral Gyrus & 6 & 418 & -34 & -11 & 36 & 3.637 & $<0.001$ \\
\hline Left Superior Temporal Gyrus & 22 & 398 & -58 & -47 & 15 & 3.837 & $<0.001$ \\
\hline \multicolumn{8}{|l|}{ Control participants } \\
\hline Right Cuneus & 18 & 617 & 17 & -92 & 15 & -3.87 & $<0.001$ \\
\hline
\end{tabular}

3

4

5

6

7

8 


\section{Figure 2}

Brain activity detected by the ASMR-eliciting $>$ control video contrasts

The results in parts (A), (B), and (C) show the responses of ASMR participants while viewing ASMR-eliciting > control videos. The peak intensity coordinates for each significantly increased cluster were located in the right superior frontal gyrus, dorsal anterior cingulate gyrus, and precentral gyrus, and the left cuneus, medial frontal, precentral, and superior temporal gyri. (A) Sagittal view. (B) Coronal view. (C) Transverse view. The results in parts (D), $(E)$, and (F) depict the contrast showing responses of the control participants while viewing ASMR-eliciting > control videos. Control participants showed significantly decreased activity in the right cuneus while watching the ASMR-eliciting videos, as compared to watching the control videos. (D) Sagittal view. (E) Coronal view. (F) Transverse view. Contrasts are displayed at $p<0.01$, cluster threshold corrected for multiple comparisons. $\mathrm{SAG}=$ sagittal, $\mathrm{COR}=$ coronal, $\mathrm{TRA}=$ transverse $\mathrm{A}=$ anterior, $\mathrm{P}=$ posterior, $\mathrm{R}=$ right, $\mathrm{L}=$ left. 
A

A

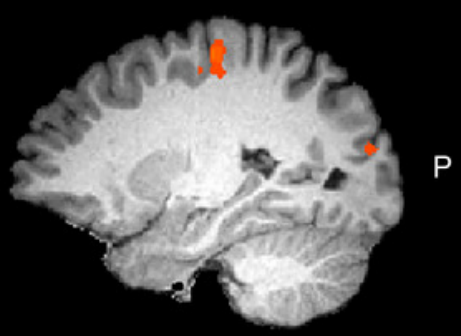

SAG

D

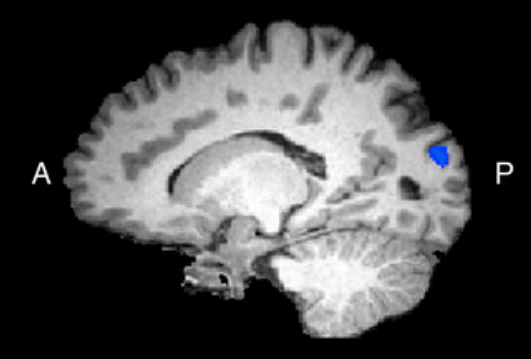

SAG
B

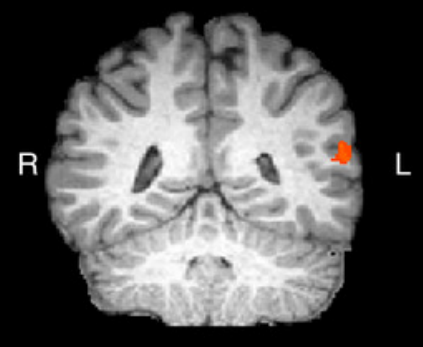

COR

E

$\mathrm{R}$

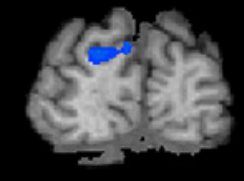

COR

L
C

R

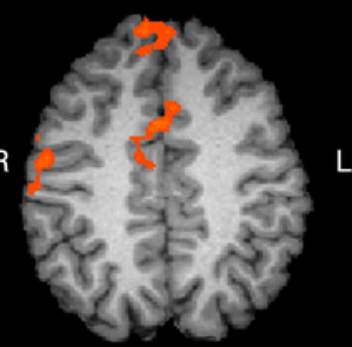

TRA

F

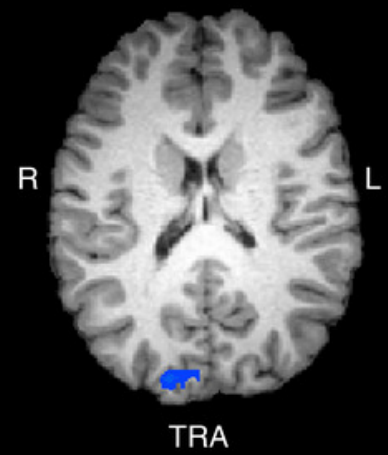

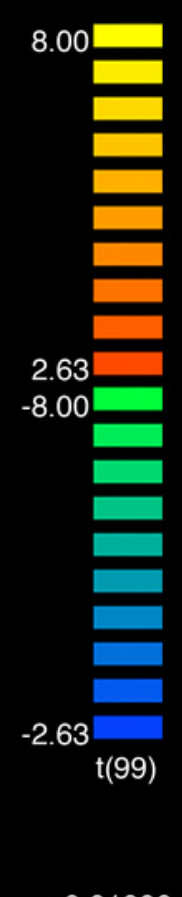

$\mathrm{p}<0.01000$ 


\section{Figure $\mathbf{3}$ (on next page)}

Beta values for the ASMR-eliciting > control video contrasts

Beta values for the within-subjects contrasts of ASMR-eliciting videos > control videos for the ASMR group (the left section of the plot) and for the control group (right side of plot). Video type is depicted in red (ASMR-eliciting) and green (control videos), with boxes indicating the interquartile medial range for beta values, whiskers indicating maximum and minimum beta values (excluding outliers). The median beta value is represented by the horizontal line and the mean value by the diamond within each box. Individual beta values per cluster are represented by black circles. The anatomical label for each cluster is shown along the bottom, for reference with Table 2 . 


\section{Table 3 (on next page)}

Coordinate and statistical values for the contrast of ASMR participants > Control participants in response to ASMR-related videos.

Positive $t$-values indicate regions of increased activity for ASMR participants and negative $t$ values indicate regions of decreased activity for ASMR participants, as compared to control participants in response to watching ASMR-related videos. 
2 Table 3. Coordinate and statistical values for the contrast of ASMR participants $>$ Control participants in 3 response to ASMR-related videos.

\begin{tabular}{|c|c|c|c|c|c|c|c|}
\hline \multirow[b]{2}{*}{ Region label } & \multirow[b]{2}{*}{ Brodmann Area } & \multirow{2}{*}{$\begin{array}{l}\text { Cluster size } \\
\text { (\# voxels) }\end{array}$} & \multicolumn{3}{|c|}{ TAL coordinates } & \multirow[b]{2}{*}{ t-value } & \multirow[b]{2}{*}{ p-value } \\
\hline & & & $\mathbf{x}$ & $\mathbf{y}$ & $\mathbf{z}$ & & \\
\hline Right Paracentral Lobule & 5 & 545 & 11 & -32 & 51 & 3.58 & $<0.001$ \\
\hline Right Cingulate Gyrus & 24 & 378 & 14 & -5 & 36 & 3.78 & $<0.001$ \\
\hline Right Thalamus & * & 356 & 8 & -23 & 15 & 2.74 & 0.007 \\
\hline Left Precuneus & 7 & 323 & -7 & -56 & 51 & 3.74 & $<0.001$ \\
\hline Left Thalamus & * & 813 & -4 & -26 & 12 & 3.22 & 0.002 \\
\hline Right Cerebellum (lingula) & * & 641 & 8 & -47 & -15 & -3.25 & 0.002 \\
\hline Left Cerebellum (culmen) & $*$ & 393 & -10 & -56 & -9 & -3.57 & $<0.001$ \\
\hline Left Cerebellum (culmen) & * & 309 & -7 & -44 & -15 & -3.13 & 0.002 \\
\hline $\begin{array}{l}4 \\
5 \\
6\end{array}$ & & & & & & & \\
\hline 7 & & & & & & & \\
\hline $\begin{array}{l}8 \\
9\end{array}$ & & & & & & & \\
\hline
\end{tabular}




\section{Figure 4}

Contrast showing responses of ASMR > control participants while viewing ASMR-related videos

Activity was increased in the right paracentral lobule, right anterior cingulate cortex, left precuneus, and bilateral thalamus, and decreased in bilateral cerebellum, for ASMR participants while viewing ASMR-related videos, as compared to the control participants watching the same videos. (A) Sagittal view. (B) Coronal view. (C) Transverse view. Contrast is displayed at $p<0.05$, cluster threshold corrected for multiple comparisons. SAG $=$ sagittal, $\mathrm{COR}=$ coronal, $\mathrm{TRA}=$ transverse $\mathrm{A}=$ anterior, $\mathrm{P}=$ posterior, $\mathrm{R}=$ right, $\mathrm{L}=$ left.

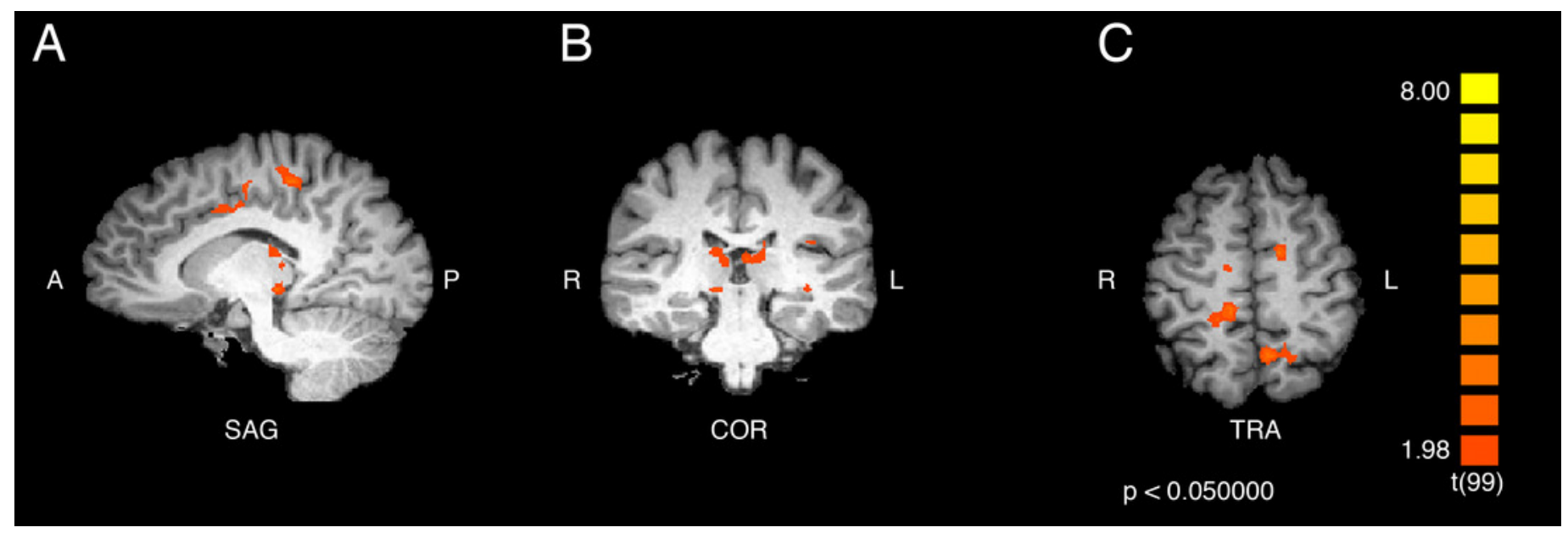




\section{Figure $\mathbf{5}$ (on next page)}

Beta values of the contrast between ASMR participants and control participants while watching the ASMR-eliciting videos.

Beta values for the between-subjects contrasts of ASMR participants as compared to control participants while watching the ASMR-eliciting videos. ASMR participants are depicted in red and control participants in green. The boxes indicate the interquartile medial range for beta values, while whiskers indicate maximum and minimum beta values (excluding outliers). The median beta value is represented by the horizontal line and the mean value by the diamond within each box. Individual beta values per cluster are represented by black circles. The anatomical label for each cluster is shown along the bottom, for reference with Table 3. 
